\title{
IX.
}

\section{Statistische Uebersicht îber die vom 1. November 1874 bis 1. November 1877 in der Berliner Universitätspoli- klinik für Ohrenkranke untersuchten und behandelten Kranken, nebst Bemerkungen zur praktischen Ohrenheilkunde.}

\author{
Yon \\ Prof. Dr. August Lucae.
}

Am 1. November 1874 wurde an der Berliner Universität eine Poliklinik für Ohrenkrankheiten in's Leben gerufen und mir die Direction derselben anvertrant. Zum Assistenten wurde Herr Dr. Dennert ernannt, weleher dureh seine bald vierjährige praktische Thätigkeit an der jetzigen Anstalt sich ein bleibendes Verdienst um dieselbe erworben hat. ${ }^{1}$ ) Ihm verdanke ich auch nachfolgende statistische Tabelle der poliklinischen Krankenbewegung. Dieselbe konnte der Natar der Sache nach nur mit annäbernder Genanigkeit gegeben werden, da unsere Poliklinik bisher kein eigenes Local besitzt, sondern dasjenige der medicinischen Universitätspoliklinik (Ziegelstrasse 8/9) benutzt. Da aber letztere von $12 \mathrm{Uhr}$ ab bis spät in die Nachmittagsstunden Kranke annimmt, so bleiben für die nnserigen als zweckmässigste Zeit die Vormittagsstunden. Dieser Umstand, sowie das bekannte unregelmässige und verspätete Kommen der Kranken bringen es mit sich, dass die Notizen nicht immer mit der wünschenswerthen Genauigkeit gebucht werden konnten. ${ }^{2}$ )

1) Derselbe verlässt dieselbe am 1. November 1878 und wird durch Herrn Dr. L. Jacobson ersetzt.

2) Diese Verhältnisse werden sich in einigen Jahren günstiger gestalten. indem die Poliklinik als Theil des im Bau begriffenen neuen UniversitätsKrankenhauses ein eigenes Local besitzen wird. Mit letzterem wird laut 
Die Annahme der Kranken erfolgte während des genannten Zeitràumes 3 mal wöchentlich von 9-11 Uhr.1)

In dem angegebenen Zeitraume haben sich im Ganzen 2388 Ohrenkranke vorgestellt; 654 vom 1 . November 1874 bis ult. October 1875, 813 rom 1. November 1875 bis ult. October 1876, 921 vom 1. November 1876 bis ult. October 1877. Davon wurden;

Unmittelbar nach der Untersuchung entlassen, resp. längere

Zeit nur beobachtet . . . . . . . . . . . . 121

Geheilt entlassen . . . . . . . . . . . . . 1013

Gebessert . . . . . . . . . . . . . . . 525

Ungeheilt . . . . . . . . . . . . . . 147

Erfolg unbekannt . . . . . . . . . . . . . . 348

In Behandlung geblieben . . . . . . . . . . . . 232

Gestorben . . . . . . . . . . . . . . . . . 2

Summa: 2388

Diese 2388 Ohrenkranke zeigten 2566 verschiedene Erkrankungsformen, welche sich in folgender Weise vertheilen:

\begin{tabular}{|c|c|c|c|c|c|c|c|c|c|}
\hline & Nomen morbi & 皆 & 莺 & 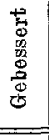 & 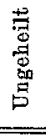 & 骂量 & 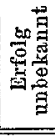 & 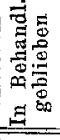 & 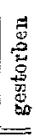 \\
\hline \multirow{13}{*}{ 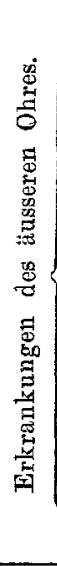 } & Lupus des Ohrläppchens & 1 & 1 & - & - & - & - & - & \\
\hline & Aspergillus . . . . . . . & 3 & 3 & 一 & - & - & - & - & \\
\hline & Verschluss d. äusseren Gehörganges & & & & & & & & \\
\hline & $\begin{array}{l}\text { a) doppelseitiger angeborener } \\
\text { b) einseitiger knöoherner dureh }\end{array}$ & 1 & — & - & 1 & - & -- & - & - \\
\hline & Eiterung . . . . . . & 1 & - & - & 1 & - & - & - & 一 \\
\hline & $\begin{array}{c}\text { Carcinom d. äusseren Gehörganges } \\
\text { ausgehend vom Felsenbein . }\end{array}$ & 1 & 一 & - & - & - & - & - & 1 \\
\hline & Traumen d. ăusseren Gehörganges & 4 & 4 & - & - & - & - & - & \\
\hline & Balggeschwulst des Ohrläppehens. & 9 & 3 & - & 一 & 6 & - & - & \\
\hline & $\begin{array}{l}\text { Cerumen: } \\
\quad \text { (einseitig 218, doppelseit. 102) } \\
\text { Ekzem: }\end{array}$ & & 320 & - & - & - & - & - & 一 \\
\hline & $\begin{array}{l}\text { (doppels. 22, einseit: 18) } \\
\text { Furunkel; }\end{array}$ & 40 & 29 & 5 & - & -1 & - & 6 & \\
\hline & (einseit. 20, doppels. 26) & 46 & 43 & - & - & -1 & - & 3 & _ \\
\hline & $\begin{array}{l}\text { Fremdkörper } \\
\text { Otitis externa diffusa: }\end{array}$ & 28 & 27 & - & - & -1 & 1 & - & \\
\hline & (einseit. 16, doppelseit. 9) . & 23 & 20 & -1 & - & 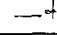 & - & 3 & - \\
\hline & Summa: & 477 & 451 & $\mathbf{5}$ & 1 & 6. & 1 & 12 & 1 \\
\hline
\end{tabular}

Ministerialbeschluss eine stationäre Klinik von 18-20 Betten verbunden sein und hierdurch einem langgefühlten Bedürfnisse abgeholfen werden, nachdem die medicinische Facultät bereits seit einer Reihe von Jahren in entgegenkommendster Weise in dieser Sache für den Verfasser dieses eingetreten war.

1) Seit dem 1. November 1877 findet die Krankenannahme 4 mal wöchentlich, Montag, Dienstag, Donnerstag und Freitag, von 9-11 Uhr statt. 


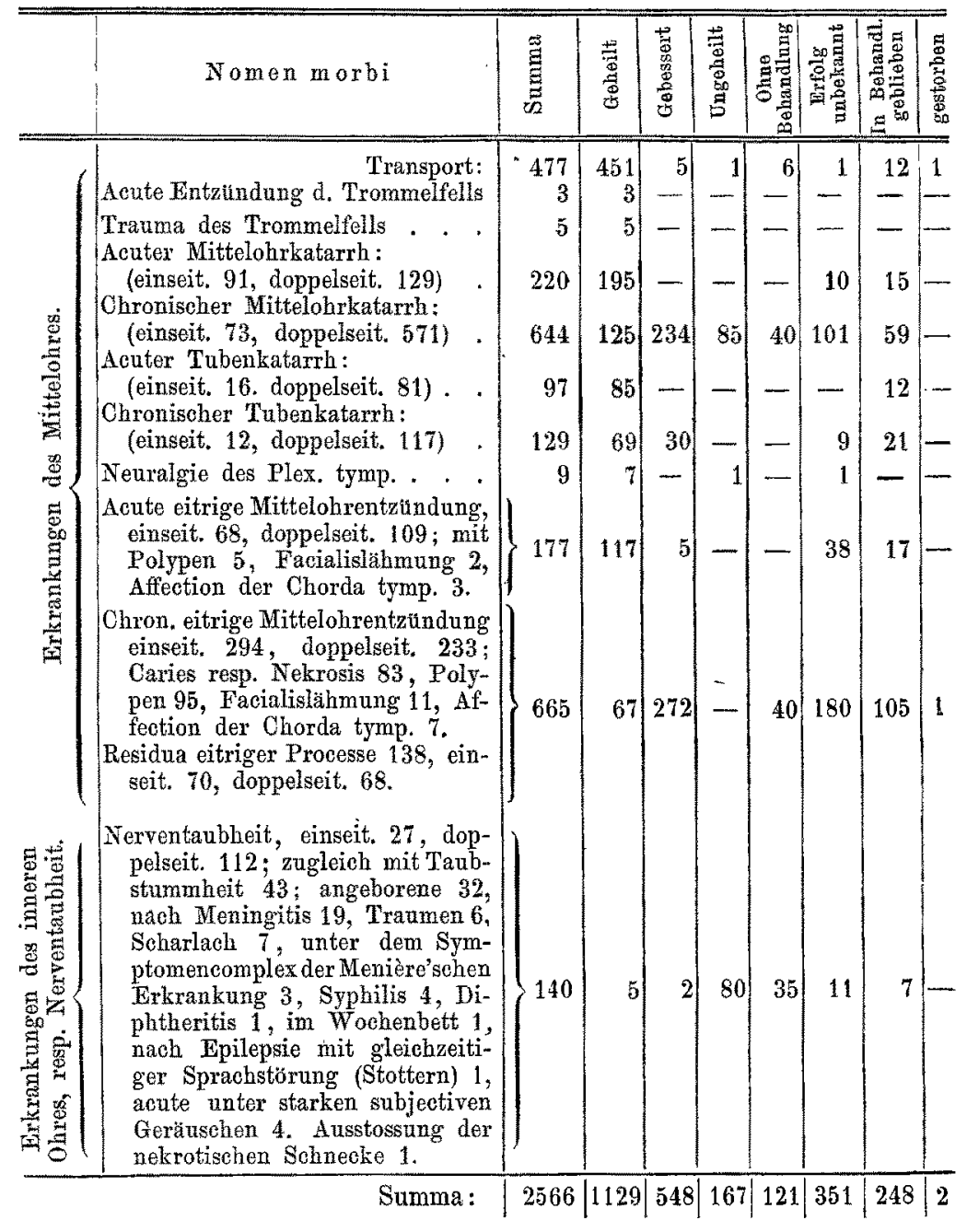

Mit Ritcksieht auf den nur sehr bedingten Werth einer poliklinischen Statistik überhaupt und wegen der, wie bereits bemerkt, nur relativen Genauigkeit des vorliegenden Berichtes liegt es mir durehaus fern, mich eingehend über den Werth der beigebrachten Zahlen zu äussern. Nur einige Punkte, welche von allgemeinem Interesse sein dürften, will ich hier hervorheben.

Was zunäehst die uablichen, auch von mir gebrauehten Ausdrïcke "geheilt, gebessert, ungeheilt" betrifft, so liegt streng. genommen nur in letzterem Worte ein zweifelloser und statistisch verwerthbarer Begriff. Die Ausdrücke "geheilt, gebessert" be- 
ziehen sich sowohl auf diejenigen Fälle, in denen gleichzeitig pathologisch-anatomisch und functionell Heilung oder Besserung nachzuweisen war, als auch auf diejenigen, in denen dieser Nachweis nur functionell oder nur pathologisch-anatomiseh möglich war. Man darf sich daher nicht wundern, dass z. B. sämmtliche 320 Fälle von Verstopfung des äusseren Gehörganges durch Cerumen "geheilt" wurden. Es ist damit nur gesagt, dass das Cerumen als fremder Körper entfernt, nicht aber, dass in sämmtlichen Fällen die Function vollkommen wieder hergestellt wurde. Aehnlich verhält es sich mit den chronischen Ohreiterungen. Unter funetionell geheilt sind aber diejenigen Kranken verstanden, welche bei ihrer Entlassung - selbstverständlich jedes Ohr für sich untersucht - schwer zu verstehende Flüsterworte, wie drei, Friedrich, Pfefferkuchen, Rauch ${ }^{1}$ ) auf wenigstens 6 Meter Entfernung sicher wiederholten. ${ }^{2}$ )

Bei der in allen Fällen sorgsam angestellten Hörprüfung und der verhältnissmässig beträchtlichen Anzahl derjenigen Fälle, in denen die eingetretene Heilung nur functionell nachweisbar - alle chronischen Mittelohrerkrankungen und die Fälle von "Nerventaubheit" bei erhaltener Continuität des Trommelfells -, dürfte es immerhin von Interesse sein, die Gesammtsumme der geheilten Fälle und speciell die Summe der geheilten Fälle genannter Kategorien mit den betreffenden Zahlen anderer grösserer Jahresberichte zu vergleichen. Ich thue dies weniger in therapentischem, als ganz besonders in diagnostischem Interesse und brauche hier erst nicht näher zu erörtern, dass die oben be-

1) Nach meiner bisherigen Erfahrung bei weitem das am schwersten zu verstehende Flisterwort.

2) Wie bereits anderwärts (dieses Arch. XII. S. 283) hervorgehoben, wird in unseren poliklinischen Journalen stets das betreffende vorgesprochene Wort hinzugefügt. Nur so ist die Prüfung der Flüstersprache praktisch und wissenschaftich verwerthbar. Will man sich -- in Folge des unter den Ohrenärzten so reichlich vertretenen Subjectivismus - um cin bestimmtes Wort (nach meinem Vorschlage "drei") durchaus nicht einigen, so sollte wenigstens in Ansehung des so verschiedenen quantitativen Werthes der einzelnen Laute und Worte endlich der mit der blossen Bezeichnung "Flüstersprache" geübte Schlendrian anfhören und Jeder der Hörweite das gebranchte Wort hinzufügen. Der von mir angegebene Phonometer kam aus Zeitmangel nur sehr selten zur Anwendung. Mag man uber die Brauchbarkeit dieses Instrumentes urtheilen wie man will: so viel ist sicher, dass dasselbe, wie ich a. a. 0 . bereits angab, gezeigt hat, dass ein gleichmässig starkes Sprechen beim Gebrauch der Flüstersprache sehr gut möglich ist. In der Poliklinik hat sich diese Thatsache $a$. A. dadurch bestatigt, dass in den Fällen, welche sowohl Herr Dr. Dennert als ich ohne Wissen des Andern auf die Flüstersprache bei Anwendung desselben. Wortes pruften, die Resultate stets fast auf 0,1 Meter ubereinstimmten. 
zeichneten Falle von Schwerhörigkeit ohne Continuitätstrennung des Trommelfells den schwächsten Punkt unsrer otiatrischen Diagnostik repräsentiren. Die unten stehende diagnostische Tabelle wird dies ohnehin zur Genüge thun.

Nach meiner reichhaltigen Beobachtung, welche sich seit einer langen Reihe von Jabren immer wieder auf diesen Punkt concentrirte, ist die Frage, ob in einem solchen Falle eine Erkrankung des Mittelohres oder Nerventaubheit vorliegt, keineswegs allein durch die übliche Untersuchung mit dem Ohrenspiegel, der Luftdonche und der Schallleitung durch die Kopfknochen zu entscheiden. Die pathologisch - anatomischen Veränderungen am Trommelfell sowie die Beobachtungen bei der Luftdouche sind nur selten dem Grade der Hörstörung proportional; die Kopfknochenleitung aber ist in den, meisten Fällen ausserordentlich unzuverlässig, da - besonders bei Anwendung tieferer Stimmgabeln - der ganze Schädel in Schwingungen gesetzt und hierdurch die Untersuchung jedes 0 hres für sich unmöglich wird.

Der Gang unserer Untersuchungsmethode war vielmehr der, dass nach Feststellung der Hörschärfe für die Flüstersprache, nach Benutzung des Ohrenspiegels und des - wo dies immer, auch bei Kindern, nur möglieh - Katheters überall, wo die Luftdouche negativen Erfolg hatte, eine eingehende Prüfung auf verschieden hohe Töne mittelst starker Stimmgabeln rom Contra $G$ bis $c^{4}$ in Octavenspannungen vorgenommen wurde. Seltener kamen die König'schen Stahlcylinder und andere hier nicht näher zu erörternde akustische Hilfsmittel zur Anwendung.

Es wurde hierbei die iiberraschende Beobachtung gemacht, dass eine Reihe von Erkrankungen, welche dem objectiven Befunde und der itblichen Terminologie gemäss unter " chronischer Mittelohr-, resp. Paukenkatarrh" zu registriren gewesen wären, sich als ausgesprochene Erkrankungen des inneren Ohres herausstellten, da sich bei der Untersuchung eine grosse Verschiedenheit in der Perception der hohen und tiefen Töne und zwar vorwiegend eine auffallend gute, zum Sprachverständniss umgekehrt proportionale Pereeption der tieferen Töne ergab. Die Zahl der Erkrankungen des inneren Ohres würde auf Kosten der chronischen Mittelohrerkrankungen voraussichtlich noch grösser ausgefallen sein, wenn nicht im Drange der Arbeit oder durch Wegbleiben der Kranken sich mancher Fall der genaueren Untersnchung entzogen hätte. 
Statistische Uebersicht d. Berliner Universitätspoliklinik f. Ohrenkranke. 125

Diagnostische Tabelle.

\begin{tabular}{|c|c|c|c|c|c|c|c|}
\hline \multirow[t]{2}{*}{ Beobachter } & \multirow{2}{*}{$\begin{array}{c}\text { Dauer } \\
\text { der Beob- } \\
\text { achtung }\end{array}$} & \multirow{2}{*}{ 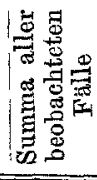 } & \multicolumn{2}{|c|}{$\begin{array}{l}\text { Chron, Erkrankung des } \\
\text { Mittelohres ohne Per- } \\
\text { foration des Trommel- } \\
\text { felles }\end{array}$} & \multicolumn{2}{|c|}{$\begin{array}{l}\text { Nerven- } \\
\text { taubheit }\end{array}$} & \multirow{2}{*}{$\begin{array}{c}\text { Ort } \\
\text { der Beob- } \\
\text { achtung }\end{array}$} \\
\hline & & & absolnt & $\begin{array}{c}\text { auf } 100 \mathrm{der} \\
\text { gesammten } \\
\text { Fälle }\end{array}$ & absolnt & $\begin{array}{c}\text { anf } 100 \text { der } \\
\text { gesammiten } \\
\text { Făllo }\end{array}$ & \\
\hline Grmiber & 3 Jahre & 2621 & 634 & 24,2 & 362 & 13,8 & Wien. \\
\hline Lucae & 3 Jahre & 2566 & 773 & 30,1 & 140 & 5,5 & Berlin. \\
\hline Schwartze & 6 Jahre & 1043 & 198 & 19,0 & 55 & 5,3 & Halle a/S \\
\hline Hedinger $\left.{ }^{1}\right)$ & 4 Jahre & 3679 & 1082 & 29,4 & 156 & 4,2 & Stuttgart. \\
\hline Poorten $^{1}$ ) & 3 Jahre & 1040 & 265 & 25,5 & 27 & 2,6 & Riga. \\
\hline
\end{tabular}

Therapeutische Tabelle.

\begin{tabular}{l|c|c|c|c}
\hline Beobachter & $\begin{array}{c}\text { Summe aller } \\
\text { wirklioh behandelten } \\
\text { Fälle }\end{array}$ & $\begin{array}{c}\text { Summe der Falle von ohron. Er- } \\
\text { krankung des Mittelohres ohne } \\
\text { Perforation des Trommelfells } \\
\text { und von Nerventaubheit }\end{array}$ \\
\cline { 2 - 5 } & behandelt & geheilt & behandelt & geheilt \\
\hline \hline & 2445 & $1129=46,2 \%$ & 838 & $199=23,7 \%$ \\
Gucae & 2245 & $1540=68,6 \%$ & 676 & $147=21,7 \%$ \\
Schwor & 964 & $499=51,8 \%$ & 211 & $30=14,2 \%$ \\
Poorten & 961 & $438=45,4 \%$ & 229 & $22=9,9 \%$ \\
Hedinger & 3284 & $1383=42,1 \%$ & $?$ & $?$
\end{tabular}

Das fremde Material zu rorstehenden 2 Tabellen habe ich vorwiegend den von mir über die Jahre 1866-1877 angefertigten otiatrischen Berichten (in den Jahresberichten ïber die Leistungen und Fortschritte der gesammten Medicin) entnommen. $\mathrm{Nur}$ in einigen Fällen lagen mir bei meiner jetzigen Arbeit die Originaltabellen vor, so dass ich für Vollständigkeit und vollkommene Richtigkeit der Zahlen nicht überall einstehen kann. Im Uebrigen habe ich keine Miuhe gescheat, die grösste Genanigkeit zu erzielen.

Bei Aufstellung der Vergleichsstatistik sind die aus dem einen ins andere Jahr übertragenen Kranken in Abrechnung gebracht worden, sowohl in Bezug auf die Gesammtsumme, als in Bezug auf die einzelnen Fälle von n chronischen Mittelohrerkran-

1) Es ist hervorzuheben, dass diese beiden Autoren nicht speciell uber poliklinische, sondern über die von ihnen überhaupt behandelten Obrenkranken berichten. 
kungen ohne Perforation des Trommelfells" und "Nerventaubheit". Unter ersteren sind sämmtliche Fälle von nicht eitrigen chronischen Erkrankungen des Mittelohres, der Tuba E. und der Paukenhöhle, unter letzteren alle diejenigen Fälle von Schwerhörigkeit rubrificirt, deren Sitz nach Angabe der Beobachter jenseits der Paukenhöhle in centripetaler Richtung zu suchen ist.

In der therapeutischen Tabelle sind selbstverständlich die nicht behandelten Fälle von der Gesammtsumme der Fälle abgezogen worden.

Man ersieht aus der diagnostischen Tabelle, dass die Gruber'sche Statistik an Zahl der Fälle von "Nerventaubheit" alle übrigen sprungweise übertrifft, während die Hedinger'sche die kleinste Zahl aufweist. Abgesehen von dem bereits oben erwähnten Umstande, dass unsere Diagnostik in Fällen ohne Perforation des Trommelfells noch so häufig dem subjectiven Ermessen des einzelnen Beobachters unterworfen ist, duirften vielleicht (?) klimatische Verhältnisse auf die Zahl der chronischen Mittelohrerkrankungen ohne Perforation des Trommelfells (resp. Katarrhe) und auf die Zahl der Fälle von "Nerventaubheit" einen wesentlichen Einfluss üben. Ich habe aus diesem Grunde auch den Ort des Beobachters hinzugefügt.

Was die therapentische Tabelle betrifft, so habe ich wegen des höchst unbestimmten Begriffes "gebessert" die Zahl der gebesserten Fälle ausgeschlossen. Aber auch über die der ${ }_{n}$ geheilten" Fälle enthalte ich mich weiterer Erörterung. Die Zahlen mögen selbst reden und dazu anregen, bei späteren statistischen Uebersichten den Begriff "geheilt" genau za präcisiren.

Aus den speciellen, in der statistischen Uebersicht unserer diesjährigen poliklinischen Thätigkeit angeführten Erkrankungen sei zunächst die auffallend geringe Zahl (3) der Fälle von Aspergillus des äusseren Gehörganges hervorgehoben. Dieselbe erklärt sich zum Theil aus dem Charakter einer Poliklinik, welche nicht immer die zur genauen Diagnose nothwendige mikroskopische Untersuchung gestattet, andererseits jedoch aus der seit über 10 Jahren von mir gemachten Erfahrung, dass die Otomycosis vorwiegend eine Krankheit der wohlhabenderen Klasse ist und daher häufiger in der Privatpraxis zur Behandlung kommt. -

Der Fall von angeborenem Verschluss des äusseren Gehörganges betraf ein neugeborenes Kind. Er zeichnet sich durch den doppelseitigen Verschluss des Ohres aus; ferner dadurch, 
dass die fast immer gleichzeitige Verkümmerung der Ohrmuschel nur auf einer Seite vorhanden war, während auf der anderen die Ohrmuschel vollkommen normal war.

Von den mit Caries des Felsenbeines einhergehenden chronischen Mittelohreiterungen seien drei erwähnt. Zunächst die beiden Todesfälle. Der eine Fall wurde sehliesslich, da sich drohende Allgemeinerscheinungen zeigten, in einem hiesigen Krankenhause aufgenommen und ging daselbst durch Caries und Thrombose des Sinus transversus zu Grunde. Gelegenheit zur Section wurde uns nicht gegeben. Der andere, ein Fall von primärem Epithelialcareinom des Felsenbeines, verdient wegen der noch immer geringen Anzahl der Beobachtungen ein besonderes Interesse, und gebe ich daher folgende, meinen Notizen wörtlich entnommene Krankengeschichte:

Am 6. Nor. 1874 stellte sich in meiner Privatsprechstunde der 37 jährige Postsecretär Habermann aus Berlin vor. Blasse Gesichtsfarbe. Seit Kindheit Ausfluss aus dem rechten Ohre. Flistersprache: R. am Ohre (vierzehn), L. normal. Im rechten äusseren Gehörgang stinkende Eitermassen und "polypöse", von der vorderen Wand des knöchernen Gehörganges ausgehende Wucherungen.

11. Nov. Entfernung von 3 erbsengrossen "Polypen “. Aufnahme in der Poliklinik, in der er sich am

9. Februar 1875 einfand. Die Polypen wieder gewachsen, galvanokaustisehe Operation derselben.

12. Febr. Zu Hause hat sich noch ein Stïek von der Grösse einer kleinen Kirsche abgestossen. Salicyllösung zum Ausspritzen 1 auf 400 .

16. Febr. Schmerzen in der Umgebung des Ohres. Tinet. Jodi zum Einpinseln.

19. Febr. Recidiv der Wueherung. Schwellung des knorpligen Gehörganges, Incision in denselben.

3. März. Nach wiederholter galvanokaustischer Operation ist der "Polyp" wieder gewachsen und füllt den Gehörgang vollständig aus. Die Schmerzen constant. Der Kranke macht zum ersten Mal einen bedenklichen Eindruck. Sehr anffallend ist die dureh Betheiligung des Kiefergelenkes hervorgerufene Verschiebung des Unterkiefers nach links, welche anfangs eine Facialislähmung vortäuschte. Die Untersuchung eines Theiles der Geschwulstmasse des äusseren Gehörganges (Dr. O rth) lässt es zweifelhaft, ob ein Sarkom vorliegt.

Es wird dem Kranken gerathen, sich in die Charité anfnehmen zu lassen, was er nach langem Zögern wegen der heftigen Schmerzen endlieh that.

Am 6. März entfernte Herr Geh.-Rath Bardeleb en unter CarbolNebel mit Messer, seharfem Löffel und Finger sämmtliche Geschwulstmassen bis zur cariösen Paukenböhte (das Trommelfell schien ganz zu Grunde gegangen zu sein), nachdem zuvor ein ergiebiger, vom 
Tragus nach dem Jochbein gehender Schnitt gemacht war, theils um den Gehörgang zu erweitern, theils um die Parotis frei zu legen. Letztere stellte sich hierbei als völlig intakt heraus. Die leicht zerreisslichen gelb-röthlichen. Geschwulstmassen - im Ganzen etwa vom Volumen einer Kirsche - wurden sofort einer genauen mikroskopischen Untersnchung unterworfen und zeigten das charakteristische Bild dés Epithelialkrebses, indem sich uberall die bekannten mit - zum Theil verhornten - Epithelien angefiillten Nester nachweisen liessen. Herr Dr. Orth hatte die Güte, die betreffenden Objecte zu controliren. Pat. fühlte sich nach der Operation beträchtlich erleichtert and verliess aach 21 Tagen "geheilt* und ohne Schmerzen die Charité. Erst am 1. Juni 1875 fand sich Pat. wieder in der Poliklinik ein. Derselbe bot jetzt sowohl in Bezug auf den örtlichen als allgemeinen Befund einen im höchsten Maasse trostlosen Anblick dar (s. die Abbildung).

Die ganze rechte Ohrgegend ist im Umfange einer Faust vorgewölbt. Vor der Ohrmuschel eine grosse ulcerirende flache Geschwulst, welche aus zwei von einander durch eine Wand geschiedene kraterförmige Geschwiire gebildet wird, von

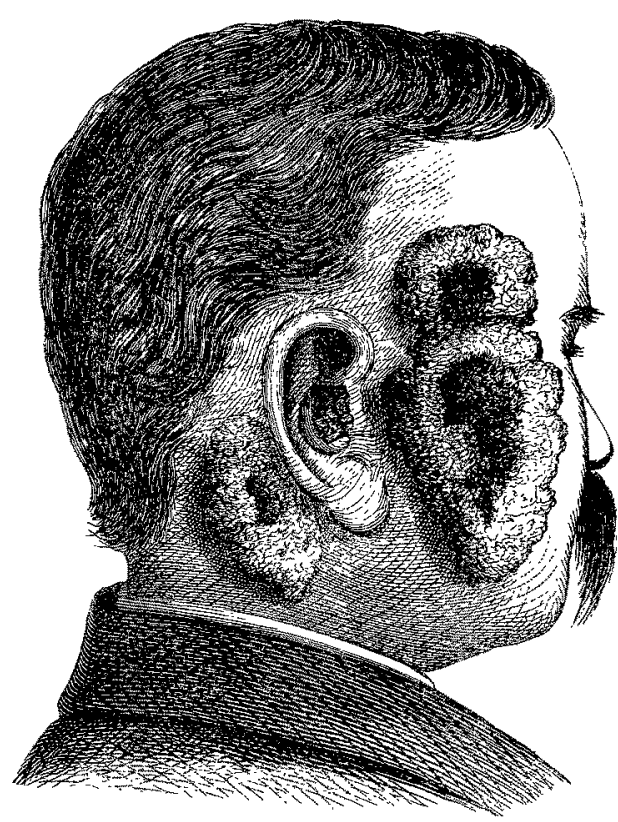
mehr als doppelter Grösse der Ohrmuschel. Hinter dem Ohre eine zweite ulcerirende Geschwalst, welche bis unterhalb des Ohrläppchens herabzieht, von der Grösse zweier Wallnuisse. Die im äusseren Gehörgang befindliche Geschwulst ist nicht beträchtlich grösser als vor ihrer operativen Entfernung.

Beim Einspritzen ins Ohr dringt das Wasser durch sämmtliche Geschwürsöffnungen vor und hinter dem Ohre hervor. Der rechte weiche Gaumen steht tiefer und mehr nach vorn vorhängend als der linke. Fast totale rechtseitige Facialisläh mung. Allgemeine Kachexie mit hektischem Fieber ; constante furibunde Schmerzen.

Von einer weiteren operativen Behandlung konnte hier keine Rede sein. Herr Dr. Dennert widmete dem Unglücklichen noch einige Zeit seinen ärztlichen Beistand. Nach langem Zureden liess Pat. sich endlich wieder in die Charité aufnehmen, ohne dass wir den Termin seiner Aufnahme in Erfahrung brachten. Bei meiner 
Rïekkehr von der Ferienreise erfuhr ich nachträglich, dass Pat. bereits am 14. September 1875 verstorben war, so dass ich der am 15. September stattgehabten Section leider nicht habe beiwohnen können. Da die Section in die Ferien fiel, war anch kein eingehendes Protokoll aufgenommen und erhielt ich von Herrn Dr. Orth, welcher die Section gemacht, nur die rverzeichnete pathologisch-anatomische Diagnose: Carcinoma ulcerosum capitis. Meningitis purulenta dextra. Perforatio ossis petrosi dextri. Carcinoma et perforatio durae matris, abscessus gangraenosus lobi temporal. dextri. Herr Dr. Orth theilte mir noch mündlich mit, dass der durch die Paukenhöhlendecke exfolgte Durchbruch von der Grösse eines Markstückes gewesen sei. Das Präparat war nicht aufgehoben worden.

Der Fall gleicht in vieler Beziehung dem von Schwartze in diesem Archiv Bd. IX. beschriebenen Fall von primärem Epithelialkrebs des Mittelohres, zeichnet sich jedoch von diesem besonders dadurch ans, dass der Warzenfortsatz lange Zeit hindurch völlig intact schien. Anch in dem Schwartze'schen Falle hatte seit Kindheit eitriger Ausfluss bestanden. Ioh stimme diesem Beobachter darin bei, dass mancher Fall von Epithelialkrebs des Mittelohres uns bisher entgangen sein mag. Die frühzeitige mikroskopische Untersuchung dürfte jedoch, wie mein Fall lehrt, nicht immer die Diagnose vollkommen sicher stellen. Es schien anfangs zweifelhaft, $o b$ es sich wirklich um einen malignen Tumor handelte. Jedenfalls erweckte die mikroskopische Untersuchung in mir gründliches Misstrauen gegen die bis dahin gebuchte Diagnose "Ohrpolyp" und stand ich sofort von der weiteren galvanokaustischen Operation ab, die möglicher Weise nur zum schnelleren Wachsthum der Geschwulst beigetragen hat. - Noch besonders hervorzuheben ist, dass letztere nicht von der Parotis ausging.

Ein erfreulicheres - wenn auch in Bezug auf die Function immerhin erbarmungswerthes - Bild zeigt folgender Fall von chronischer Ohreiterung mit Caries des Felsenbeines. Aus der Reihe analoger Fälle, in denen die Anbohrung, resp. Ausräumung des cariösen Warzenfortsatzes vorgenommen wurde, hebe ich diesen hier besonders hervor, weil in ihm kurze Zeit nach der Operation eine vorher bestandene Facialislähmung zur vollkommenen dauernden Heilung kam:

21. Mai 1875. Adolph Schwalezyk, 11/3 Jahr, aus Berlin, kam als schwächliches Kind mit ausgesprochenem specifischem Hautaussehlag zur Welt. Seit 5 Monaten beiderseits Ohrenfluss. Das anscheinend völlig taube Kind zeigt beiderseits Otitis media purulenta 
granulosa mit Perforation des Trommelfells. Hinter dem linken Ohre eine Fistel, durch welehe die Sonde auf ranhen Knochen stösst.

1. Juni. Ausgesprochene Facialislähmung. Die Oeffung hinter dem Ohre wird erweitert und darauf der Warzenfortsatz mit dem scharfen Löffel ausgeräumt. Zum Verband: Charpie mit Acid. salicyl. 0,1 , Glycerini 10,0 .

8. Juni. Die Facialislähmung völlig verschwanden.

11. Juni. In der Tiefe jetzt ein grosser Sequester sichtbar, welcher am

7. December entfernt wird. Es stellt derselbe einen beträcht lichen Theil des Warzenfortsatzes dar.

24. Mai 1876. Wunde hinter dem Ohre verheilt, an Stelle derselben eine tiefe Grube.

11. October. Es zeigt sich in der Narbe wieder eine stecknadelkopfgrosse Oeffnung. Cupr. sulfocarbol. 0,5, Ung. Glycerini 25,0 zum̀ Verband.

26. Juni 1877. Die Oeffnung hinter dem Ohre völlig geschlossen durch eine im Durchmesser $1 \frac{1 / 2}{\mathrm{Cm}}$. und $1 \mathrm{Cm}$. tiefe Knochennarbe. Facialislähmung hat sich nicht wieder gezeigt. Beiderseits, namentlich rechts, noch leichte Ohreiterung, gegen welche Einpulverungen 'von Alum. usti, Acid. salicyl. ana und Solut cupr. sulfocarbol. $0,3: 30,0$ abwechselnd angewendet werden.

Wenn auch in diesem Falle die operative Eröffnnng und Austäumung des cariösen Warzenfortsatzes wegen der sofort eintretenden dauernden Heilung der Facialislähmung and der wesentlichen Besserung der Ohreiterung den besten Erfolg hatte, wenn ferner durch die allgemeine Behandlung des Pat. (Jod, Eisen, Leberthran, Bäder etc.) das Aussehen desselben sich wesentlich besserte, so ist die Gehörlosigkeit in Nichts geändert und ist der nunmehr 4 jährige Knabe taubstumm. Jedenfalls liegen noch tiefere Störungen im inneren Ohre, resp. das vor, was ich durch obigen ganz allgemeinen Begriff "Nerventaubheit" bezeichnet habe: Es sind also damit bei unserer grossen Unsicherheit der Diagnose, namentlich in Fällen von gänzlicher Taubheit Veränderungen zu verstehen, welche im Labyrinthe oder im Gehörnerven oder im Gehirn ihren Sitz haben. Als ätiologiseh wichtig sei noch erwähnt, dass eingestandner Maassen vor und während der Geburt des Kindes Ulcera specifica matris a patre translata bestanden.

Der zuletzt beschriebene Fall führt uns schliesslich zur Klasse der "Nerventaubheit".

Was die 5 als geheilt aufgefuhrten Fälle anlangt, so waren dies 3 Fälle, in denen starke subjective Geräusche das hervorragendste Symptom bildeten; ron den 2 anderen war der eine 
durch Syphilis, der andere nach Trauma entstanden. Die beiden gebesserten beziehen sich anf zwei im Scharlach erworbene Affectionen (des Labyrinthes?).

In Bezug auf die Therapie und in specie die der subjectiven Gehörsempfindungen seien hier nur zwei häufig von uns angewandte Mittel erwähnt. Einmal das von Wo a k e ư̛̆d He $\mathrm{m}$ ming ${ }^{1}$ ) gegen pulsirende subjective Ohrgeräusche empfohlene Acid. hydrobromicum, welches in einige en Fällen - dreimal täglieh 15 bis 20 Tropfen in concentrirtem Zuckerwasser - einen eclatanten Nutzen zeigte, im Uebrigen jedoch nicht mehr zu leisten sehien, als das Kal. bromatum. Das andere, ein mechanisches Hülfsmittel bestand in der zeitweise in der Poliklinik mit Hülfe des gewöhnlichen Gummiballons vorgenommenen starken Luftverdichtung im äusseren Gehörgange. ${ }^{2}$ ) Die Wirkung war stets schnell voriibergehend; die Kranken - und zwar eine nicht geringe Anzahl - gaben jedoch auf das Bestimmteste an, dass während des einige Sekunden anhaltenden Druckes die Geränsche vollkommen schwanden. Das Auffallendste aber war, dass dies einige Mal in denselben Fällen beobachtet wurde, in denen die gewöhnliche Luftdouche von der Nase aus Linderung der subjectiven Geräusche hervorbrachte. Es liegt auf der Hand, dass nach diesen Beobachtungen die Labyrinthdruck-Theorie der subjectiven Gehörsempfindungen auf höchst schwankendem Boden steht. Die Meinung aber, dass diejenigen Falle, in denen die Luftverdünnug im äusseren Gehörgange die subjectiven Geräusehe momentan herabsêtze, für die Tenotomie des Tens. tympani "wirklich geeignet" seien, muss hiernach als eine vollkommen haltlose bezeichnet werden. ${ }^{3}$ )

Aus der Klasse der Fälle von "Nerventaubheit " seien schliesslich noch zwei interessante Fälle angeführt, welche ein Bild geben von unserer in dergleichen Fällen geübten Untersuchungsmethode:

Bäcker Flaschmann, 59 Jahre alt, aus Berlin, kam am 17. Nov. 1876 zur Beobachtung. Starker Mann. Seit 12 Jahren schwerhörig, seit 2-Jahren Schwindelerseheinungen und Sausen, welches in letzter Zeit nachgelassen hat. - Der Kranke kann nicht mit geschlossenen Augen vorwärts gehen, er schwankt sofort. Pat. anscheinend völlig

1) Med. Press and circul. Aug. 1, 1877.

2) Das olivenförmige Nasenstück des Ballons wird zur besseren luftdichteren Adaptirung am zweckmässigsten vorher in Wasser getaucht und fest ins $\mathrm{Ohr}$ eingesetzt. Den Drnck steigere man vorsichtig and allmählich. 3) Näheres hierüber im Jahresbericht der gesammten Medicin für 1877 . Bd. II. S. 483 . 
taub, kann nicht lesen. Er sieht jedoeh gut von den Lippen ab. An beiden Trommelfellen keine auffallenden Veränderungen. Pat. kann nur rechts einsilbige laut gesprochene Zahlen auffassen (die Zahlen 1,2, schwieriger 14), Die vorgesprochenen Vocale sagtier nicht nach. Dasselbe viel schlechter (die Zahlen 6 und 4 uñsher) links. - Ein derartiger Kranker muss dem Ohrenart jede Frage erst nachsprechen, damit man gewiss ist, dass er dieselbe verstanden bat. - Seit einem halben Jahre hat das Gesicht allmählich abgenommen in demselben Grade wie das Gehör. Bei der (unter genauer Controle) mit Stimmgabeln, resp. König'schen Stahlcylindern (von $e^{5} a b$ ) vorgenommenen Untersuchung hört Pat.: Contra-G r. als Brummen, l. nicht:

$\mathrm{C}$ ebenfalls nur rechts.

c und $c^{1}$ ebenso.

$c^{2}$ nur rechts "ganz fein ".

$\mathrm{c}^{3}$ beiderseits Null, desgleichen $\mathrm{c}^{4}, \mathrm{c}^{5}, \mathrm{~g}^{5}, \mathrm{e}^{6}, \mathrm{~g}^{6}, \mathrm{c}^{7}$. Stimmgabel $\mathrm{e}^{3}$ hört er per Kopf knochen nicht.

24. November 1876. Hat nach dem das vorige Mal ord. Chin. sulf. (0,5 pro dosi et die) kein stärkeres Sausen bekommen. Auscultation per Kath.: Tuba beiderseits frei, freies Blasegeräusch. Hört $\mathbf{c}^{3}$ mit Resonator nicht.

1. December. Pat. hört nicht die in jedes Ohr besonders gesprochenen Consonanten $\mathrm{s}$, sch, wohl aber räth er Worte, in denen diese vorkommen, wie "Fleisch, sieben ". Ebenso bei b (Brod). Statt "Zwieback" antwortet er "Kuhdreck". F als Silbe (ef) spricht er nach, den Laut $f$ nicht, dagegen spricht er "Pferd" nach. $H$ nicht, statt "Galgen ": „Besen". Statt des Lautes h "Buch". Statt „Hammel" "Bild". Auch das hohe vordere eh hört er nicht. Ebenfalls das tiefe-hintere eh und $\mathrm{r}$ nicht. - Kal. jodati $2: 200$.

5. December. Per Kopfknochen Contra-G, C, e, e $\mathrm{e}^{1}$ nur von den Zähnen wahrgenommen (als Brummen?). Immer noch Schwindel. 29. Januar 1877. Angeblich nach elektrischer Behandlung (Dr. Remak) schlechter.

23. März. Nach 16maliger galvanischer Behandlung hat sich in Bezug auf Gehör und Schwindel nichts geändert, Sausen soll nur noch bei Anstrengungen, Niesen ete. eintreten.

ohne weitere Belandlung entlassen.

Dem vorliegenden wichtigen Falle von "Nerventaubheit", deren Sitz - was wenigstens das rechte Ohr betrifft - wohl zweifellos ins Labyrinth żu verlegen ist, möchte ich noch einige nabeliegende Bemerkungen hinzufügen.

Vor Allem ist hervorzuhebeu, dass Pat. bei genauerer, auf die einzelnen Töne und Spràchlaute gerichteten Functionsprüfung (stets bei geschlossenen Augen) links absolut Nichts, rechts dagegen nur die Töné vom Contra- $\mathrm{G}-\mathrm{e}^{1}$ deutlich, das $\mathrm{c}^{2}{ }_{n}$ ganz fein", demnach undeutlich hörte, während er noch - namentlich 
rechts - im Stande war, einzelne Worte hin und wieder "mit dem Ohre" richtig aufzufassen. Es liegt der Schluss nahe, dass er diese Worte mittelst der sensibeln Nerven der Obrmuschel fühlte - eine Ansicht, die durch genate in dieser Richtung anzustellende Untersuchungen an Taubstummen leicht zu controliren ist. Bei meinen wiederholten Bestuchen im hiesigen Taubstummeninstitut im Jahre 1860 machte mich der damalige intelligente Director Reimer darauf aufmerksam, dass das Gefuhl bei- Tanbstummen in höchst frappanter Weise für das fehlende Gehör vicariirend eintrete, welcher Umstand oft zur Verwechselung mit angeblicher Heilung führe und den Charlatanen - wie er in einem Falle selbst nachgewiesen - Gelegenheit böte, die Taubstummen "hörend" zu machen. Er stellte mir als Beleg hierfür einen etwa 7 jährigen israelitischen Taubstummen mit lebhaften, klugen Augen vor, welcher die Lautsprache recht gut sprach. Der völlig gehörlose Knabe reagirte selbst nicht auf die heftigsten, hinter seinem Rïcken in einiger Entfernung hervorgebrachten Geräusche. - Schloss er die Augen fest zu, und sprach man ihm in die Volae beider auf den Rücken neben einander gelegten Hände mässig laut aber langsam und deutlich Worte hinein, welche - wie "Papa“, "Berlin" ete. - seinem Ideenkreise angehörten, so wiederholte er sie schnell und sicher. Der Versuch wurde so oft, und auch mit anderen Worten wiederholt, dass von einer Tänschung keine Rede sein konnte. -

Interessant ist ferner die - von uns wie bereits oben gesagt, auch in anderen Fällen häufig beobachtete - Tontaubheit für die hohen und höchsten Töne rechterseits; und ist hierbei hervorzuheben, dass selbst das stärkste Ansehlagen der so schrill klingenden Stahlcylinder sowie die Gabel $\mathrm{e}^{3}$ mit dazu gehörigem Resonator nicht wahrgenommen wurde. Das grösste Gewicht aber möchte ich auf die Beobachtung legen, dass Pat. auf dem rechten Ohre nicht einmal den Vocal $U$ hörte, obwohl er die tiefen, der $U$-Lage entsprechenden Töne vom Contra- $G$ bis zum c $^{t}$ sicher "wahrnahm".

Diese Erscheinung erklärt sich vielleicht so, dass er eben der höheren zum charakteristischen U-Klange nothwendigen Partialtöne ${ }^{1)}$ verlustig gegangen war, oder - was mir wahrscheinlicher - auch dadurch, dass er die ihm noch übrig gebliebenen

1) Vgl. Helmholtz, Tonempindungen. 3. Ausgabe. S. 187. 
tiefen Töne gar nicht mehr als solche, sondern vielleicht nur als unbestimmte Geräusche hörte. Für letztere Annahme spricht wenigstens der Umstand, dass Schwerhörige, wenn auch in geringerem Grade als der in Rede stehende Kranke, so häufig die ihnen vorgesprockenen, einfachen Worte mit anderen, sowobl dem Sinne als dem Klange nach durchaus heterogenen Worten verwechseln, und führt dies zu dem naheliegenden Schluss, dass auch die bei der Untersuchung gebrauchten Töne der Stimmgabeln, das klingende Geräusch der Uhr etc. oft qualitativ verändert aufgefasst werden wird.

Als praktisch wichtig geht aber hieraus hervor, dass bei einer exacten mit letzteren Instrumenten vorgenommenen Prufung die Kranken ebenfalls angeben mïssten, was sie eigentlich hören. Da dies aber in beschränkter Weise nur bei Anwendung von Tönen und von musikalischen Kranken zu erwarten, so bleibt als alleiniges Prüfungsmittel von sicherem, allgemeinem Werthe nur die Sprache ibrig. ${ }^{1}$ )

Den Schluss dieses Berichtes mag der von Herrn Dr. Dennert in meiner Abwesenheit beobachtete Fall von "Nerventaubheit" naeh Epilepsie bei gleichzeitiger Sprachstörung bilden:

Wilhelm Richter, 30 Jahre, Ackerer aus Kleden bei Lübbenau. Hat angeblich vor 3 Wochen nach starker Erhitzung beim Mähen kaltes Wasser getrunken und sich dann auf die Erde gelegt, worauf er eine Art Krämpfe bekam, wobei ihn 5 Mann gehalten baben sollen. Die Krämpfe kamen nach seiner Schilderung nicht zum Ausbruch und sollen danach Störungen im Gehör und in der Sprache eingetreten sein, welche allmählich zugenommen haben. Pat. will friber immer gut gebört haben. Beiderseits Sausen und Klingen. Keine Schwindelerscheinungen.

R. lant geschrien a. 0 . (30).

L. $" n, "(20)$.

Aufgefordert "ein Wort auszusprechen, gelingt ihm dies erst nach längerer Anstrengung, und nachdem die Mundöffnung und die Stellung der Kiefer gegeneinander nach verschiedenen Richtungen hin krampfhaft verzogen worden sind, und wenn er dabei gegen den Unterkiefer fest andrückt. Stört man ihn darin, den Unterkiefer zu unterstïtzen, so muss er noch grössere Anstrengungen machen, um das Wort herauszubringen und kommt dasselbe mehr flïsternd und ohne Brustton heraus.

Trommelfell rechts stark eingezogen, Hammergriff perspektivisch verkïrzt, sonst die Farbe des Trommelfelles ziemlich normal.

Links das Trommelfell noch in höherem Grade eingezogen, in Bezug anf seine Farbe ebenfalls wesentlicher als auf der anderen Seite verändert.

1) Vgl. dieses Archiv Bd. XII. S. 282. 
Kath.: Tuba beiderseits durchgängig, weiches Geräusch. Nach der Luftdonche in Bezug auf das Gehör keine Aenderung. Stimmgabeltöne werden bis zum $\mathrm{c}^{4}$ beiderseits stark aber gleichmässigherabgesetzt vernommen und zwar werden sie links besser percipirt wie rechts. Die König'schen Stäbe werden in demselben Verhältniss herabgesetzt vernommen. Ord.: Kal. brom. 20,0:300,0.

14. Angust 1877. Pat. stösst beim Sprechen jetzt nur noch wenig an und ist nicht mehr genöthigt, den Unterkiefer dabei zu unterstützen. Hört jetzt beiderseits Fluistersprache a. 0.1) Pat wird der elektrischen Behandlung unterworfen. (Gonstanter Strom, Anodenbehandlung des oberen Nackens.)

17. Angust. Spricht jetzt schon fast ohne Anstossen und hört die Flüstersprache schon etwas vom Ohre entfernt.

21. August. Pat. spricht normal und hört Flïstersprache beiderseits ca. 0,7 .

24. August. Pat. hat angeblich in Folge davon, dass er den Kopf auf den linken Vorderarm zum Ausruhen gelegt hatte, eine Lähmung desselben bekommen. Er ist nicht im Stande, mit seiner Hand einen starken Druck anszuiuben und die Hand gegen die Dorsalfläche des Vorderarmes zu erheben.

26. August. Gehör und Sprache fast zur Norm zurückgekehrt. Die Lähmung des linken Vorderarmes beginnt sich zu bessern.

28. August. Ans der Behandlung entlassen mit fast normalem Gehör, Mangel jeder Sprachstörung und Restitution der Lähmung des linken Vorderarmes ad integrum.

Zur Epikrise sei kurz erwähnt, dass nach Ansicht des Herrn Dr. Remak, weleher auf Veranlassung des Herrn Dr. Dennert die - 14 tägige - elektrische Behandlung gütigst übernahm, die im vorliegenden Falle gleichzeitig beobachtete Sprachstörung eine "Mischung von Stottern und Scandiren" war. Mit Rücksicht auf das frühere gute Hörvermögen des Pat. und den verhältnissmässig negativen Befund im äusseren und mittleren $\mathrm{Ohr}$ (Trommelfelltribung und Einziehung) warde die Diagnose auf "Nerventaubheit" gestellt und dieselbe bei der gleichzeitigen Sprachstörung als eine centrale angesprochen. Wie weit dem constanten Strome oder dem gleichzeitigen Gebrauch des Bromkalium in Betreff der in kurzer Zeit erzielten Heilung die Palme gebührt, bleibt unentschieden.

Der Fall von Ausstossung der nekrotischen Schnecke ist bereits in diesem Archiv Bd. X. von Herrn Dr. Dennert mitgetheilt worden.

1) i. e. am Ohr. Wo in meinen Journalen kein Wort bei Fl. (= Flüstersprache) in Klammern steht, ist stets die Zahl 3 gemeint. 